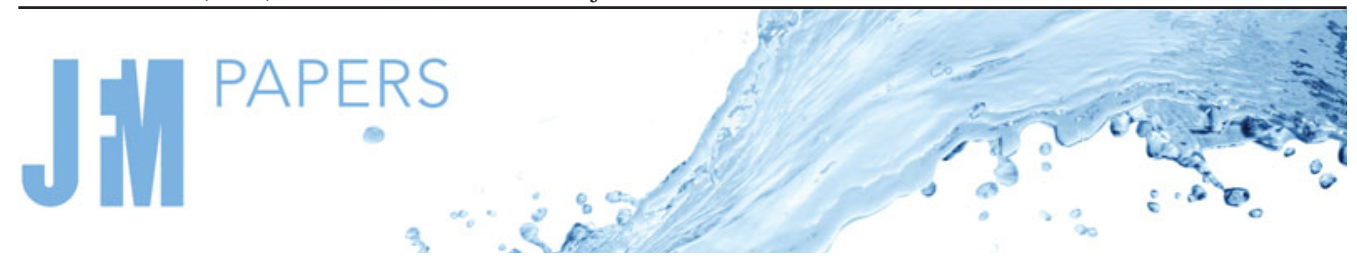

\title{
On the amplitude and the flow force constant of steady water waves
}

\author{
Evgeniy Lokharu $\dagger$ \\ Department of Mathematics, Linköping University, SE-581 83 Linköping, Sweden
}

(Received 30 March 2021; revised 30 March 2021; accepted 22 May 2021)

We prove a new explicit inequality for the non-dimensional flow force constant, significantly improving the Benjamin and Lighthill conjecture about irrotational steady water waves. As a corollary, we prove a bound for the wave amplitude in terms of the Bernoulli constant. We show that the amplitude decays as $r^{-2}$ when $r \rightarrow+\infty$, where $r$ is the non-dimensional Bernoulli constant. We explain that the latter limit corresponds to deep water waves and the bound for the amplitude is sharp. In terms of physical parameters the result states that the amplitude $a$ of an arbitrary Stokes wave is bounded by $\mathrm{Cm}^{2} \mathrm{~g} / \mathrm{Q}^{2}$, where $m$ is the relative mass flux, $g$ is the gravitational constant, $Q$ is the total head and $C$ is an absolute constant given explicitly. In particular, this implies that $a<C c^{2} g^{-1}$, where $c$ is the wave speed. The latter inequality is valid for all Stokes waves, irrespective of wavelength or amplitude, including extreme waves.

Key words: surface gravity waves

\section{Introduction}

We consider the classical problem for irrotational steady waves on the surface of an ideal fluid of finite depth. The fluid occupies an infinite region with a rigid flat bottom and is separated from the air by an unknown free surface. For plane steady waves there are three important physical quantities: $Q, m$ and $S$. Here $Q$ is the total head, $m$ is the mass flux and $S$ is the flow force constant. The importance of these parameters was justified by Benjamin \& Lighthill (1954) in their study of long waves. It was shown that a combination of $Q, m$ and $S$ determines the cnoidal wave-train uniquely. Furthermore, non-dimensional quantities $Q / Q_{c}$ and $S / S_{c}$ of an arbitrary cnoidal wave realise points in a two-dimensional cuspidal region as in figure 1 whose boundary corresponds to laminar flows. Thus, Benjamin and

$†$ Email address for correspondence: evgeniy.lokharu@liu.se

(C) The Author(s), 2021. Published by Cambridge University Press. This is an Open Access article, distributed under the terms of the Creative Commons Attribution licence (http://creativecommons.org/

licenses/by/4.0/), which permits unrestricted re-use, distribution, and reproduction in any medium, provided the original work is properly cited. 


\section{E. Lokharu}

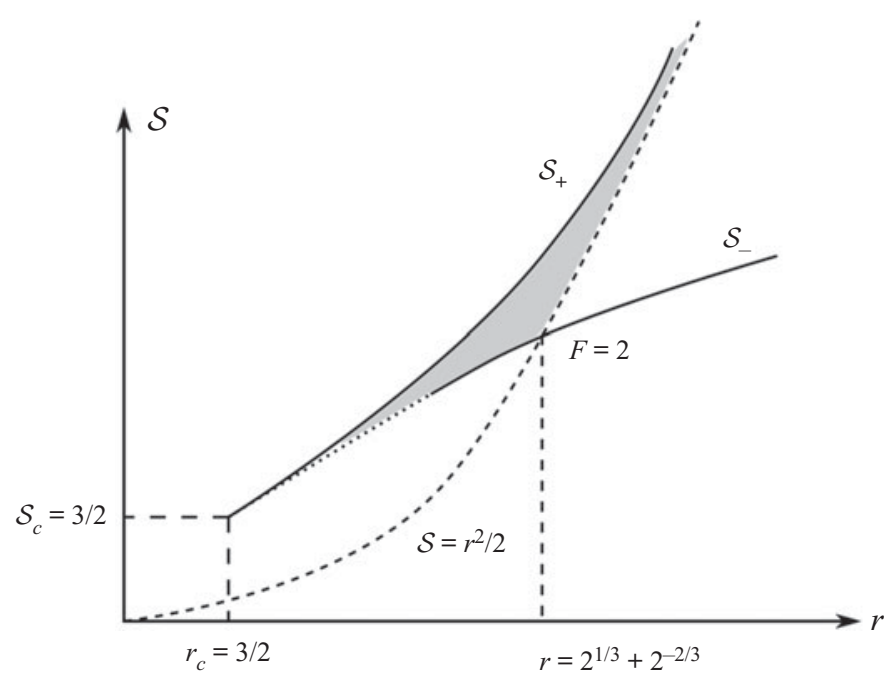

Figure 1. Parameter region for steady water waves.

Lighthill conjectured the latter property to hold among all exact gravity waves, irrespective of amplitude or wavelength.

In order to reformulate this conjecture in a mathematically convenient way we introduce non-dimensional variables proposed by Keady \& Norbury (1978), where the mass flux and the gravitational constant are scaled to unity. In new variables, constants $Q$ and $S$ are scaled $r$ and $\mathcal{S}$ respectively. According to conjecture, every steady flow, irrespective of amplitude or wavelength, will realise a point within a certain region in the $(r, \mathcal{S})$-plane. The latter planar region is determined by all points $(r, \mathcal{S})$ for which

$$
\mathcal{S}_{-}(r) \leq \mathcal{S} \leq \mathcal{S}_{+}(r),
$$

where $\mathcal{S}_{+}(r)$ and $\mathcal{S}_{-}(r)$ are the flow force constants corresponding to the subcritical and supercritical laminar flows respectively. The boundary of this region is a cusped curve representing all uniform streams (see figure 1). Thus, the conjecture can be stated as follows.

Benjamin and Lighthill Conjecture. For any two-dimensional steady wave the corresponding constants $r$ and $\mathcal{S}$ are subject to the inequalities (1.1).

We emphasise that the conjecture concerns all steady waves, not necessarily periodic or solitary waves. For instance, sub-harmonic bifurcations were found numerically by Vanden-Broeck (1983). Such waves can have several crests in every minimal period.

The conjecture restricted to Stokes waves (periodic waves with exactly one crest and one trough in every minimal period) was verified by Benjamin (1995), although the left inequality in (1.1) was obtained earlier by Keady \& Norbury (1975). Later, Kozlov \& Kuznetsov $(2009,2011)$ proved (1.1) for arbitrary solutions under weak regularity assumptions, provided the Bernoulli constant $r$ is close to its critical value $r_{c}=3 / 2$. It was extended to the rotational setting in Kozlov, Kuznetsov \& Lokharu (2017), again for $r \approx r_{c}$, which guarantees that solutions are of small amplitude. The left inequality in (1.1) for periodic waves with a favourable vorticity was obtained by Keady \& Norbury (1978). Finally, a complete answer was given in Lokharu (2020), where the conjecture was proved in its general form for rotational waves with an arbitrary vorticity distribution.

Another important question about the conjecture is whether all points of the region between curves $\mathcal{S}_{-}$and $\mathcal{S}_{+}$in figure 1 correspond to some steady motion. It was already 
noted by Benjamin \& Lighthill (1954) that the answer is negative and that the diagram should be complemented by the third barrier corresponding to waves of extreme form. This was partly approved by Cokelet (1977) who numerically found the barrier, which, however, consists of waves that are only close to stagnation but not extreme.

In the present paper we prove a new and surprisingly simple inequality $\mathcal{S}>r^{2} / 2$ that is valid for all steady waves, irrespective of geometry and amplitude. The new inequality corresponds to a barrier defined by the curve $\mathcal{S}=\frac{1}{2} r^{2}$ that intersects the lower boundary at a point with $F=2$ (recovering the well-known bound for the Froude number) and is asymptotically close to the upper boundary $\mathcal{S}=\mathcal{S}_{+}(r)$. This shows that all steady waves, including waves of greatest height, correspond to a significantly smaller part (grey region in figure 1) of the region determined by the inequalities (1.1).

In addition to the significant improvement in the Benjamin and Lighthill conjecture, the new result can be used to study solutions of the problem for large $r$. More precisely, we prove that all steady waves with large Bernoulli constants are necessarily small in their amplitude, which is of order $O\left(r^{-2}\right)$. Thus, every extreme wave with the mass flux $m=1$ in water of depth $d$ has the amplitude of order $1 / d^{2}$. In the physical variables the result states that the amplitude $a$ of an arbitrary Stokes wave is bounded by $\mathrm{Cm}^{2} \mathrm{~g} / Q^{2}$, where $m$ is the relative mass flux, $g$ is the gravitational constant, $Q$ is the total head and $C$ is an explicit absolute constant.

\section{Statement of the problem}

We consider the classical model for two-dimensional steady waves on water of finite depth. We neglect the effects of surface tension and consider an ideal fluid of constant (unit) density. In the corresponding moving reference frame stationary Euler equations are given by

$$
\begin{gathered}
(u-c) u_{X}+v u_{Y}=-P_{X}, \\
(u-c) v_{X}+v v_{Y}=-P_{Y}-g, \\
u_{X}+v_{Y}=0 \\
u_{Y}-v_{X}=0,
\end{gathered}
$$

which holds true in a two-dimensional fluid domain $D_{\eta^{\star}}$, defined by the inequality

$$
0<Y<\eta^{\star}(X) \text {. }
$$

Here $(u, v)$ are components of the velocity field, $Y=\eta^{\star}(X)$ is the surface profile, $c$ is the wave speed, $P$ is the pressure and $g$ is the gravitational constant. The corresponding boundary conditions are

$$
\begin{gathered}
v=0 \text { on } Y=0, \\
v=(u-c) \eta_{X}^{\star} \text { on } Y=\eta^{\star}, \\
P=P_{a t m} \text { on } Y=\eta^{\star} .
\end{gathered}
$$

We reformulate these equations in terms of a stream function $\psi^{\star}$, defined implicitly by the relations

$$
\psi_{y}^{\star}=c-u, \quad \psi_{x}^{\star}=v .
$$

This determines $\psi^{\star}$ up to an additive constant, while relations $(2.1 f),(2.1 f)$ require $\psi^{\star}$ to be constant along the boundaries. Thus, by subtracting a suitable constant we assume that

$$
\psi^{\star}=m^{\star}, \quad Y=\eta^{\star} ; \quad \psi^{\star}=0, \quad Y=0 .
$$




\section{E. Lokharu}

Here $m^{\star}$ is the mass flux, defined by

$$
m^{\star}=\int_{0}^{\eta^{\star}}(c-u) \mathrm{d} Y
$$

The corresponding problem for the stream function is

$$
\begin{gathered}
\Delta \psi^{\star}=0 \text { in } D_{\eta^{\star}}, \\
\frac{1}{2}\left|\nabla \psi^{\star}\right|^{2}+g Y=Q^{\star} \text { on } Y=\eta^{\star}, \\
\psi^{\star}=m^{\star} \text { on } Y=\eta^{\star}, \\
\psi^{\star}=0 \text { on } Y=0 .
\end{gathered}
$$

In the following we will use the non-dimensional variables proposed by Keady \& Norbury (1978), where the gravitational constant and the relative flux are scaled to unity. More precisely, we put

$$
\begin{aligned}
& x=\lambda X, \quad y=\lambda Y, \quad \eta(x)=\lambda \eta^{\star}\left(\lambda^{-1} x\right), \quad \psi(x, y)=\left(m^{\star}\right)^{-1} \psi^{\star}(X, Y), \\
& r=Q /\left(m^{\star} \lambda\right)^{2},
\end{aligned}
$$

where

$$
\lambda=\left(\frac{g}{m^{2}}\right)^{1 / 3} .
$$

The corresponding non-dimensional problem is

$$
\begin{gathered}
\Delta \psi=0 \text { in } D_{\eta}=\{(x, y): 0<y<\eta\}, \\
\frac{1}{2}|\nabla \psi|^{2}+y=r \text { on } y=\eta, \\
\psi=1 \text { on } y=\eta, \\
\psi=0 \text { on } y=0 .
\end{gathered}
$$

Here $r$ is referred to as the Bernoulli constant. The above problem admits another spatial constant of motion (see Benjamin (1984) for more details), the flow force, defined as

$$
\mathcal{S}=\int_{0}^{\eta}\left(\frac{1}{2}\left(\psi_{y}^{2}-\psi_{x}^{2}\right)-y+r\right) \mathrm{d} y .
$$

After taking the $x$-derivative in (2.9) and using (2.8a) together with the boundary relation $(2.8 b)$, one verifies that $\mathcal{S}$ is independent of $x$. Our main result can now be stated as follows.

THEOREM 2.1. Let $(\psi, \eta) \in C^{2, \gamma}\left(\overline{D_{\eta}}\right) \times C^{2, \gamma}(\mathbb{R})$ for some $\gamma \in(0,1)$ be a solution to (2.8) with $\sup \eta<r$, other than a laminar flow. Then $\mathcal{S}>\frac{1}{2} r^{2}$, where $r$ and $\mathcal{S}$ are the Bernoulli and the flow force constants respectively, defined by (2.8b) and (2.9). 
By laminar flows we mean a solution independent of the $x$-variable. Let us compare the quantity $\mathcal{S}_{+}(r)$ and the bound $\frac{1}{2} r^{2}$ from the theorem. Note that

$$
\mathcal{S}_{+}(r)=\frac{1}{2 d_{+}(r)}-\frac{1}{2} d_{+}^{2}(r)+r d_{+}(r)
$$

where $d_{+}(r)>1$ (the depth of the subcritical laminar flow) is the unique root of $\frac{1}{2} d^{-2}+$ $d=r$. Thus, for large $r$, we find $d_{+}(r) \sim r$ and more precisely,

$$
d_{+}(r)=r-\frac{1}{2 r^{2}}+O\left(r^{-3}\right) \text { as } r \rightarrow+\infty .
$$

Therefore, we obtain

$$
\begin{aligned}
\mathcal{S}_{+}(r) & =\frac{1}{2 r}\left(1+O\left(r^{-3}\right)\right)-\frac{1}{2}\left(r^{2}-\frac{1}{r}+O\left(r^{-2}\right)\right)+r\left(r-\frac{1}{2 r^{2}}+O\left(r^{-3}\right)\right) \\
& =\frac{r^{2}}{2}+\frac{1}{2 r}+O\left(r^{-2}\right) .
\end{aligned}
$$

We see that the curve $\mathcal{S}=\frac{1}{2} r^{2}$ is below the upper boundary $\mathcal{S}=\mathcal{S}_{+}(r)$ (see figure 1) for large $r$ and is asymptotically accurate. On the other hand, curves $\mathcal{S}=\mathcal{S}_{-}(r)$ and $\mathcal{S}=$ $\frac{1}{2} r^{2}$ have one point of intersection, for which the Froude number $F:=d^{-3 / 2}=2$. Indeed, because

$$
\mathcal{S}_{-}(r)=\frac{1}{2 d_{-}(r)}-\frac{1}{2} d_{-}^{2}(r)+r d_{-}(r),
$$

the equality $\mathcal{S}_{-}(r)=\frac{1}{2} r^{2}$ is equivalent to $\left(r-d_{-}\right)^{2}=d_{-}^{-1}$, which in view of $r=\frac{1}{2} d_{-}^{-2}+$ $d_{-}$, leads to $d^{-3 / 2}=2$. This recovers the well known bound $F<2$ for the Froude number of solitary waves; see Starr (1947), Amick \& Toland (1981), McLeod (1984).

Using the result of Theorem 2.1 we can prove the following statement.

THEOREM 2.2. There exists a constant $C>0$ such that the inequalities

$$
\max \eta-\min \eta<C r^{-2} \text { and } 0<\psi_{y}<C r^{-1}
$$

hold true for any Stokes wave solution in $D_{\eta}$. The constant $C$ is independent of $r$.

Let us discuss the result, which might look surprising. First, we consider a sufficiently deep water wave $\eta^{\star}$ with $c^{2} /(g d)$, being small, where $d$ is the average water depth, being large. Then $m^{\star} \approx c d$ and $Q^{\star} \approx g d$, so that $r \approx\left(c^{2} /(g d)\right)^{-1 / 3}$ is large. If $a^{\star}$ is the amplitude of $\eta^{\star}$, then the corresponding amplitude of $\eta$ is

$$
a=a_{\star} O\left(r^{-2}\right)
$$

which has the same order as the bound in Theorem 2.1. Thus, deep water waves correspond to $(\psi, \eta)$ with large $r$, while the opposite is also true in view of $(2.14 a, b)$. Therefore, our result is sharp for deep water waves. 


\section{E. Lokharu}

Comparing variables, we find the corresponding inequality for the amplitude $a$ in the original physical variables:

$$
a<C \frac{1}{\lambda r^{2}}=C \frac{m^{2} g}{Q^{2}} .
$$

On the other hand, we have $m \leq c d$ and $Q \geq g d$, so that

$$
a<C c^{2} g^{-1} \text {. }
$$

This inequality is valid for all Stokes waves, irrespective of wavelength or amplitude, including extreme waves. This is confirmed numerically in several studies, such as Cokelet (1977) and Thomas (1975).

The result of Theorem 2.2 has another important meaning. We can imagine two extreme Stokes waves with $m=1$ and corresponding mean depths $d_{1}=10$ and $d_{2}=1000$. The first wave has an amplitude of order $10^{-2}$, while for the second it is of order $10^{-6}$. Now we can consider two Stokes waves with the same depths and the same amplitude, of order $10^{-2}$. Then the underlying flows of such waves must be very different and the mass flux of the second wave corresponding to the depth $d_{2}$ must be significantly greater. In other words, the wave with depth $d_{2}$ must be travelling much faster. Of course, this observation is also true for usual Stokes waves without stagnation points. This resembles the well known property of surface waves.

\section{Proof of Theorem 2.1}

\subsection{Flow force function formulation}

Based on the definition for the flow force constant (2.9), we introduce the corresponding flow force function

$$
F(x, y)=\int_{0}^{y}\left(\frac{1}{2}\left(\psi_{y}^{2}\left(x, y^{\prime}\right)-\psi_{x}^{2}\left(x, y^{\prime}\right)\right)-y^{\prime}+r\right) \mathrm{d} y^{\prime} .
$$

Just as in Basu (2020) we can reformulate the water-wave problem in terms of the function $F$. It is straightforward to obtain

$$
F_{x}=\psi_{x} \psi_{y}, \quad F_{y}=\frac{1}{2}\left(\psi_{y}^{2}-\psi_{x}^{2}\right)-y+r
$$

Thus, we arrive at an equivalent formulation given by

$$
\begin{gathered}
\Delta F+1=0 \text { for } 0<y<\eta, \\
\frac{1}{2}\left(-\eta^{\prime} F_{x}+F_{y}\right)+y=r \text { on } y=\eta, \\
F=\mathcal{S} \text { on } y=\eta, \\
F=0 \text { on } y=0 .
\end{gathered}
$$

A reformulation as given above was already covered in Constantin \& Strauss (2011) with an arbitrary vorticity. This reformulation is useful for an analysis of solutions with weak regularity. 
First, we show that $F>0$ for $y>0$. In fact, one can verify that

$$
F_{y} \geq \psi_{y}^{2}>0 \text { for } 0 \leq y \leq \eta \text {. }
$$

It is enough to apply the maximum principle to the subharmonic function $\Phi=\frac{1}{2}\left(\psi_{x}^{2}+\right.$ $\left.\psi_{y}^{2}\right)+y$. A direct calculation gives

$$
\Delta \Phi=\psi_{x x}^{2}+2 \psi_{x y}^{2}+\psi_{y y}^{2} \geq 0
$$

while $\Phi_{y}=1$ on $y=0$. Thus, by the maximum principle, the maximum of $\Phi$ is attained at the surface, where $\Phi=r$ is constant. This shows that $0<\Phi<r$ in $D_{\eta}$. Using this in $(3.2 a, b)$ we obtain (3.4).

Using equations for the flow force function $F$ one can obtain an interesting formula for the flow force constant $\mathcal{S}$ of a Stokes wave. For this purpose we apply Green's first identity to find

$$
\int_{-\Lambda}^{\Lambda} \int_{0}^{\eta(x)}\left\{\left(\Delta F \cdot y+F_{y}\right\} \mathrm{d} x \mathrm{~d} y=\int_{-\Lambda}^{\Lambda} \eta(x)\left(F_{x}(x, \eta(x)), F_{y}(x, \eta(x))\right) \cdot\left(-\eta^{\prime}(x), 1\right) \mathrm{d} x .\right.
$$

Here $\Lambda$ is the half period, while $\eta(x)$ is even with respect to $x$. Thus, using $(3.2 a, b)$ and (3.3) we conclude

$$
2 \mathcal{S} \Lambda-\frac{1}{2} \int_{-\Lambda}^{\Lambda} \eta^{2} \mathrm{~d} x=2 \int_{-\Lambda}^{\Lambda} \eta(r-\eta) \mathrm{d} x
$$

Therefore, we obtain

$$
\mathcal{S}=\frac{1}{2 \Lambda} \int_{-\Lambda}^{\Lambda}\left(2 r \eta-\frac{3}{2} \eta^{2}\right) \mathrm{d} x \text {. }
$$

This equality is similar to Starr's identity for the Froude number of a solitary wave; see Starr (1947).

In what follows it is convenient to have the 'mass flux' $F=\mathcal{S}$ scaled to 1 . This suggests new variables

$$
X=\mathcal{S}^{-1 / 2} x, \quad Y=\mathcal{S}^{-1 / 2} y, \quad \zeta(X)=\mathcal{S}^{-1 / 2} \eta(x), \quad \bar{F}(X, Y)=\mathcal{S}^{-1} F(x, y) . \quad(3.9 a-d)
$$

The scaled problem is

$$
\begin{gathered}
\Delta \bar{F}+1=0 \text { for } 0<Y<\zeta, \\
\frac{1}{2}\left(-\zeta_{X} \bar{F}_{X}+\bar{F}_{Y}\right)+Y=R:=r \mathcal{S}^{-1 / 2} \text { on } Y=\zeta, \\
\bar{F}=1 \text { on } Y=\zeta, \\
\bar{F}=0 \text { on } Y=0 .
\end{gathered}
$$

Furthermore, in view of (3.4), we additionally have

$$
\inf \bar{F}_{Y}>0 \text {. }
$$

Note that $\psi_{y}$ is always separated from zero for any smooth steady wave as in Theorem 2.1.

We are going to prove certain bounds for the Bernoulli constant $R$ in (3.10b). Note that the system (3.10) is very similar to the stream-function formulation of the water problem with constant vorticity, for which the desired bounds were obtained in Kozlov, Kuznetsov \& Lokharu (2015). Thus, a similar argument can be applied here and we adapt it below. 


\section{E. Lokharu}

\subsection{Stream solutions}

In order to obtain bounds for $R$ we need to study stream solutions to (3.10). These are pairs $\bar{F}=U(Y ; d), \zeta(X)=d$, parametrised by the depth $d>0$. Using this ansatz in $(3.10)$, one finds

$$
U(Y ; d)=-\frac{1}{2} Y^{2}+\left(d^{-1}+\frac{1}{2} d\right) Y .
$$

The corresponding Bernoulli constant is given by

$$
R(d)=\frac{1}{2 d}+\frac{3 d}{4}
$$

In view of $(3.10 e)$ we are interested in unidirectional solutions only, for which $U_{Y}>0$ on $[0, d]$. As a result we obtain a restriction on $d$ such that

$$
0<d<d_{0}=\sqrt{2}
$$

where the critical value $d_{0}$ is characterised by the relation $U_{Y}\left(d_{0} ; d_{0}\right)=0$, while $U_{Y}\left(Y ; d_{0}\right)>0$ for $Y \in\left[0, d_{0}\right)$. Let us put

$$
R_{0}=R\left(d_{0}\right)=\sqrt{2} \quad \text { and } \quad R_{c}=\sqrt{\frac{3}{2}}, \quad d_{c}=\sqrt{\frac{2}{3}} .
$$

Note that $R_{c}=R\left(d_{c}\right)$ is the global minimum of $R(d)$. Thus, for any $R \in\left(R_{c}, R_{0}\right)$ there are two solutions $d=d_{-}(R)$ and $d=R_{+}(d)$ with $d_{-}(R)<d_{+}(R)$ to the equation

$$
R=R(d)
$$

For $R>R_{0}$ there is only one root $d=d_{-}(R)$. These depths are analogous to the subcritical and supercritical depths of conjugate laminar flows of the original water-wave problem.

\subsection{Bounds for the Bernoulli constant}

Our aim is to prove the following theorem:

THEOREM 3.1. Let $(\bar{F}, \zeta)$ be an arbitrary non-trivial (other than a stream) solution to (3.10). Then the corresponding Bernoulli constant $R$ is subject to the inequality $R<R_{0}$.

Note that the statement of Theorem 2.1 follows directly from the upper bound $R<R_{0}$. Indeed, a non-trivial solution $(\psi, \eta)$ of the original system (2.8) with the Bernoulli constant $r$ generates a solution to (3.10) with $R=r \sqrt{\mathcal{S}}^{-1}$. Now, Theorem 3.1 gives $r \sqrt{\mathcal{S}}^{-1}<R_{0}=\sqrt{2}$, which is equivalent to $\mathcal{S}>\frac{1}{2} r^{2}$ as stated in Theorem 2.1.

Proof of Theorem 3.1. Our argument is based on a comparison of a given solution $(\bar{F}, \zeta)$ to different stream solutions from $\S 3.2$. For this purpose we will apply the partial hodograph transformation introduced in Dubreil-Jacotin (1934) but for the flow force function formulation (3.10). This is possible because $\bar{F}_{Y}>0$ everywhere by (3.4). 
The amplitude and flow force constant of steady water waves

Thus, we introduce new independent variables

$$
q=X, \quad p=\bar{F}(X, Y),
$$

while the new unknown function $h(q, p)$ (height function) is defined from the identity

$$
h(q, p)=Y .
$$

Note that $h$ is related to the flow force function $\bar{F}$ through the formulas

$$
\bar{F}_{X}=-\frac{h_{q}}{h_{p}}, \quad \bar{F}_{Y}=\frac{1}{h_{p}},
$$

where

$$
\inf _{S} h_{p}>0 \quad \text { and } \quad \sup _{S} h_{p}<+\infty .
$$

An equivalent problem for $h$ is given by

$$
\begin{gathered}
\left(\frac{1+h_{q}^{2}}{2 h_{p}^{2}}+p\right)_{p}-\left(\frac{h_{q}}{h_{p}}\right)_{q}=0 \text { in } \Gamma=\mathbb{R} \times(0,1), \\
\frac{1+h_{q}^{2}}{2 h_{p}}+h=R \text { on } p=1, \\
h=0 \text { on } p=0 .
\end{gathered}
$$

The surface profile $\zeta$ becomes the boundary value of $h$ on $p=1$ such that

$$
h(q, 1)=\zeta(q), \quad q \in \mathbb{R} .
$$

For a detailed derivation of (3.19) we refer to Basu (2020). Applying a similar transformation for the stream functions $U(Y ; d)$, we obtain the corresponding height functions $H(p ; d)$, subject to

$$
\left(\frac{1}{2 H_{p}^{2}}+p\right)_{p}=0, \quad H(0)=0, \quad H(1)=d, \quad \frac{1}{2 H_{p}(1 ; d)}+d=R(d) . \quad(3.21 a-d)
$$

Because the domain for $h$ and $H$ is the same, we can compare these functions using the maximum principle. More precisely, we put

$$
w^{(d)}=h-H(\cdot ; d),
$$

and using the corresponding equations for $h$ and $H$ one finds that $w^{(d)}$ solves the homogeneous elliptic equation

$$
\frac{1+h_{q}^{2}}{h_{p}^{2}} w_{p p}^{(d)}-2 \frac{h_{q}}{h_{p}} w_{q p}^{(d)}+w_{q q}^{(d)}-w_{p}^{(d)}+\frac{\left(w_{q}^{(d)}\right)^{2} H_{p p}}{h_{p}^{2}}-\frac{w_{p}^{(d)}\left(h_{p}+H_{p}\right) H_{p p}}{h_{p}^{2} H_{p}^{2}}=0 .
$$

This equation can be obtained directly by substituting $h=H+w^{(d)}$ into $(3.19 b)$ and then using the corresponding equation for $H$. Thus, every $w^{(d)}$ is subject to the maximum principle; see Vitolo (2007) for an elliptic maximum principle in unbounded domains.

The proof relies on two lemmas. 


\section{E. Lokharu}

LEMmA 3.2. There exists $0<d<d_{0}$ such that $\sup _{\mathbb{R}} \zeta<d$.

Proof. Note that when $d \rightarrow d_{0}=\sqrt{2}$ we have $H_{p}(1 ; d) \rightarrow+\infty$. Thus, we can choose $d<\sqrt{2}$ for which $w_{p}^{(d)}<0$ everywhere on $p=1$. Let us show that the supremum of $w:=w^{(d)}$ in $\Gamma$ cannot be attained on $p=1$. This claim is trivial when the supremum of $w$ is attained at some point. The remaining possibility is that there exists an unbounded sequence $\left\{q_{j}\right\}$ for which $\sup _{\Gamma} w=\lim _{j \rightarrow+\infty} w\left(q_{j}, 1\right)$. Then we consider shifts $w_{j}(q, p)=$ $w\left(q-q_{j}, p\right)$ and use a compactness argument as follows. The regularity assumption from Theorem 2.1 and the relation $(3.19 a)$ guarantee that

$$
\|h\|_{C^{2, \gamma}(\bar{\Gamma})}<+\infty
$$

and so the norms $\left\|w_{j}\right\|_{C^{2, \gamma}(\bar{\Gamma})}$ are uniformly bounded. By the compactness argument there exists a subsequence $\left\{q_{j_{k}}\right\}$ and a function $w_{0} \in C^{2}(\bar{\Gamma})$ with the following property: for any compact $K \subset \bar{S}$, functions $w_{j_{k}}$ converge to $w_{0}$ in $C^{2}(K)$. Thus, the limiting function $w_{0}$ attains its global maximum at $(0,1)$, while $\left(w_{0}\right)_{q}(0,1) \leq 0$ because $w_{p}<0$ on $p=1$ by the choice of $d$. But this contradicts the Hopf lemma and then we have shown that the supremum of $w$ can not be attained for $p=1$. Now the maximum principle for $w$ forces $w \geq 0$ in $\Gamma$ and then $\zeta \leq d$. This finishes the proof of the lemma.

LEMMA 3.3. For any $q \in \mathbb{R}$ we have $\zeta(d)>d_{-}(R)$.

Proof. By Lemma 3.2 we can find $0<d_{\dagger}<d_{0}$ such that $\inf _{\mathbb{R}} \zeta=d_{\dagger}$; note that $\inf _{\mathbb{R}} \zeta>$ 0 as shown in Kozlov \& Kuznetsov (2007) (Theorem 2.3). Thus, the function $w:=w^{\left(d_{\dagger}\right)}$ is non-negative along the boundaries and so must be strictly positive in $\Gamma$ by the maximum principle. The zero infimum of $w$ is attained at the surface by the choice of $d$, so that there exists a minimising sequence $\left\{q_{j}\right\}$ such that $w\left(q_{j}, 1\right) \rightarrow 0$ as $j \rightarrow+\infty$. Then we claim that $w_{q}\left(q_{j}, 1\right) \rightarrow 0$ as $j \rightarrow+\infty$ and $\liminf w_{p}\left(q_{j}, 1\right) \leq 0$. These statements are trivial if the sequence $\left\{q_{j}\right\}$ is bounded. Otherwise we can use a similar compactness argument as in the proof of Lemma 3.2. Thus, passing to the limit in (3.19c) we obtain

$$
R=\lim _{j \rightarrow+\infty} h\left(q_{j}, 1\right)+\frac{1+h_{q}^{2}\left(q_{j}, 1\right)}{2 h_{p}^{2}\left(q_{j}, 1\right)} \geq d_{\dagger}+\frac{1}{2 H_{p}^{2}\left(1 ; d_{\dagger}\right)}=R\left(d_{\dagger}\right),
$$

which implies that $\zeta(q) \geq d_{\dagger} \geq d_{-}(R)$ for all $q \in \mathbb{R}$. The inequality here is strict until $\zeta$ is not a constant everywhere, which follows from the Hopf lemma.

Now we can prove that $R<R_{0}$. Let us choose $d_{\star}<d_{0}$ such that $\sup _{\mathbb{R}} \zeta=d_{\star}$. Just as before we can find a maximising sequence $q_{j}$, possibly unbounded, such that

$$
\lim _{j \rightarrow+\infty} \zeta\left(q_{j}\right)=d_{\star}, \quad \lim _{j \rightarrow+\infty} w_{q}^{\left(d_{\star}\right)}\left(q_{j}, 1\right)=0, \quad \lim _{j \rightarrow+\infty} w_{p}^{\left(d_{\star}\right)}\left(q_{j}, 1\right) \geq 0 . \quad(3.26 a-c)
$$

Thus, taking the corresponding limit in $(3.19 c)$ and using relations $(3.26 a-c)$ we obtain $R \leq R\left(d_{\star}\right)$. Assuming $R \geq R_{0}$ we would conclude that $d_{\star}<d_{-}(R)$, which contradicts the statement of Lemma 3.3. Therefore, we have shown $R<R_{0}$. This finishes the proof of the theorem. 


\subsection{Proof of Theorem 2.2}

First, we show that the corresponding inequality for $\psi_{y}$ is valid along the bottom. For this purpose we consider the function

$$
f=F-\mathcal{S} \psi
$$

which equals zero on $y=\eta$ and $y=0$. From (3.3a) we find that $\Delta f=-1$ in $D_{\eta}$, and so $f_{y}>0$ on $y=0$ by the maximum principle. This gives

$$
\frac{1}{2} \psi_{y}^{2}+r-\mathcal{S} \psi_{y}>0 \text { on } y=0 .
$$

Now we note that because the function $\frac{1}{2} \psi_{x}^{2}+\frac{1}{2} \psi_{y}^{2}+y$ attains its maximum at the upper boundary, we have $\frac{1}{2} \psi_{y}^{2} \leq r$ in $D_{\eta}$. Using this in (3.28), we obtain

$$
\psi_{y}<2 r \mathcal{S}^{-1}<4 r^{-1}
$$

where the right inequality is valid because $\mathcal{S}>\frac{1}{2} r^{2}$ by Theorem 2.1. The opposite inequality $\psi_{y}>r^{-1}$ for $y=0$ follows from the maximum principle applied to the function $g=\psi-r^{-1} y$, which attains its minimum on $y=0$. Thus, we have shown that

$$
r^{-1}<\psi_{y}<4 r^{-1} \text { on } y=0 .
$$

Now we are ready to prove the main claim. It is enough to show that $r-\check{\eta}<C r^{-2}$, where $\check{\eta}=\min _{\mathbb{R}} \eta$. Let us consider the function

$$
g(x, y)=-k \psi+\frac{1}{2}\left(\psi_{y}^{2}+\psi_{x}^{2}\right)+y, \quad(x, y) \in D_{\eta},
$$

where $k$ is to be determined later. A direct computation shows that

$$
\Delta g \geq 0,
$$

so that the maximum of $g$ is attained at the boundary of $D_{\eta}$. At the bottom we have

$$
g_{y}=1-k \psi_{y}>0,
$$

by (3.30), provided $k=r / 4$. Therefore, the maximum of $g$ is attained at the surface and the Hopf lemma gives

$$
g_{y}=\frac{\eta^{\prime \prime} \psi_{y}^{2}-k\left(\eta^{\prime 2}+1\right) \psi_{y}+1}{\eta^{\prime 2}+1}>0, \quad y=\eta(x) .
$$

This inequality is equivalent to

$$
2 \eta^{\prime \prime}(r-\eta)\left(1+\eta^{\prime 2}\right)^{-1}-k \sqrt{2(r-\eta)\left(1+\eta^{\prime 2}\right)}+1 \geq 0 .
$$

Let $x_{0}$ be such that $\eta^{\prime \prime}\left(x_{0}\right)=0$, then the latter inequality shows that

$$
r-\eta\left(x_{0}\right)<8 r^{-2} \text {. }
$$

Without loss of generality we can assume that $\eta(0)=\check{\eta}$. If $r-\check{\eta} \geq 16 r^{-2}$, the proof is complete. Otherwise, if $r-\check{\eta}>16 r^{-2}$ we put $\delta=r-\check{\eta}$ and note that

$$
\frac{1}{2} \delta<r-\eta(x) \leq \delta
$$

for all $x \in[0, \delta / 2]$. Here we used the fact that $\left|\eta^{\prime}\right|<1$ on $\mathbb{R}$ for any Stokes wave solution; see Strauss \& Wheeler (2016). Furthermore, $\eta^{\prime \prime}(x)>0$ on $[0, \delta]$ by (3.36). Now (3.35) 


\section{E. Lokharu}

leads to

$$
2 \delta \eta^{\prime \prime}(x) \geq k \sqrt{\delta}-1, \quad x \in[0, \delta / 2] .
$$

After integration over $[0, \delta]$, we conclude that

$$
k \sqrt{\delta}-1 \leq 4 \eta^{\prime}(\delta / 2) \leq 4
$$

Hence, $\delta<16^{2} r^{-2}$ by the choice of $k$, and we have proved that $r-\check{\eta}<16^{2} r^{-2}$. This inequality implies that $\psi_{y}<\mathrm{Cr}^{-1}$ along the surface, so that the interior estimate for $\psi_{y}$ follows from the maximum principle. This finishes the proof of Theorem 2.2.

Funding. This research received no specific grant from any funding agency, commercial or not-for-profit sectors.

Declaration of interest. The authors report no conflict of interest.

\section{Author ORCIDs.}

(1) Evgeniy Lokharu https://orcid.org/0000-0002-6781-5146.

\section{REFERENCES}

Amick, C.J. \& Toland, J.F. 1981 On solitary water-waves of finite amplitude. Arch. Rational Mech. Anal. $76(1), 9-95$.

BASU, B. 2020 A flow force reformulation for steady irrotational water waves. J. Differ. Equ. 268 (12), $7417-7452$.

Benjamin, T.B. 1984 Impulse, flow force and variational principles. IMA J. Appl. Math. 32 (1-3), 3-68.

Benjamin, T.B. 1995 Verification of the Benjamin-Lighthill conjecture about steady water waves. J. Fluid Mech. 295, 337-356.

Benjamin, T.B. \& Lighthill, M.J. 1954 On cnoidal waves and bores. Proc. R. Soc. Lond. A 224 (1159), $448-460$.

CoKelet, E.D. 1977 Steep gravity waves in water of arbitrary uniform depth. Phil. Trans. R. Soc. Lond. A 286 (1335), 183-230.

COnstantin, A. \& STRAuss, W.A. 2011 Periodic traveling gravity water waves with discontinuous vorticity. Arch. Rational Mech. Anal. 202 (1), 133-175.

DubREIL-JACOTIN, M.L. 1934 Sur la détermination rigoureuse des ondes permanentes périodiques d'ampleur finite. J. Math. Pures Appl. 13, 217-291.

KEADY, G. \& NorbuRY, J. 1975 Water waves and conjugate streams. J. Fluid Mech. 70 (4), 663-671.

KEADY, G. \& NORBURY, J. 1978 Waves and conjugate streams with vorticity. Mathematika 25 (1), 129-150.

KozLov, V. \& KuZnEtsov, N. 2007 Bounds for arbitrary steady gravity waves on water of finite depth. J. Math. Fluid Mech. 11 (3), 325-347.

KoZLOV, V. \& KuZNETSOv, N. 2009 The Benjamin-Lighthill conjecture for near-critical values of bernoulli's constant. Arch. Rational Mech. Anal. 197 (2), 433-488.

Kozlov, V. \& KuZnetsov, N. 2011 The Benjamin-Lighthill conjecture for steady water waves (revisited). Arch. Rational Mech. Anal. 201 (2), 631-645.

Kozlov, V., KuZnetsov, N. \& LOKHARU, E. 2015 On bounds and non-existence in the problem of steady waves with vorticity. J. Fluid Mech. 765, R1.

Kozlov, V., KuZnetsov, N. \& LokHARU, E. 2017 On the Benjamin-Lighthill conjecture for water waves with vorticity. J. Fluid Mech. 825, 961-1001.

LOKHARU, E. 2020 A sharp version of the Benjamin and Lighthill conjecture for steady waves with vorticity. arXiv:2011.14605.

MCLEOD, J.B. 1984 The Froude number for solitary waves. Proc. R. Soc. Edin. Sec. A 97, 193-197.

STARR, V.P. 1947 Momentum and energy integrals for gravity waves of finite height. J. Mar. Res. 6, 175-193.

STRAUSS, W.A. \& WHEELER, M.H. 2016 Bound on the slope of steady water waves with favorable vorticity. Arch. Rational Mech. Anal. 222 (3), 1555-1580.

Thomas, J.W. 1975 A numerical study of the relationship between the dimensionless parameters in the problem of periodic waves of permanent type in a liquid of finite depth. Q. Appl. Maths 32 (4), 403-410.

VANDEN-BRoeck, J.-M. 1983 Some new gravity waves in water of finite depth. Phys. Fluids 26 (9), 2385.

Vitolo, A. 2007 A note on the maximum principle for second-order elliptic equations in general domains. Acta. Math. Sin. Engl. Ser. 23 (11), 1955-1966. 